\title{
Energy switching potential energy surfaces and spectra of the van der Waals modes for the ArHCN molecule
}

\author{
A.J.C. Varandas *, S.P.J. Rodrigues, P.A.J. Gomes \\ Departamento de Química, Universidade de Coimbra, P-3049 Coimbra Codex, Portugal \\ Received 17 September 1998; in final form 2 October 1998
}

\begin{abstract}
We report fully six-dimensional potential surfaces for ArHCN by using the energy switching method. They are valid for all energy regimes and hence suitable for studying the dynamics of a wealth of processes, ranging from the dissociative reaction $\mathrm{Ar}+\mathrm{HCN} \rightarrow \mathrm{Ar}+\mathrm{H}+\mathrm{CN}$ to the rovibrational spectroscopy of the ArHCN van der Waals molecule. Calculations of rovibrational energies with $J=0$ through $J=6$ arising from $j=0$ to $j=1$ levels of HCN are also presented. (C) 1998 Elsevier Science B.V. All rights reserved.
\end{abstract}

\section{Introduction}

The rovibrational spectra and dynamics of the ArHCN van der Waals molecule has been much studied experimentally [1-8] and theoretically [4,912]. Experimentally, all studies have essentially provided information only about the angular part of the $\mathrm{Ar}-\mathrm{HCN}$ potential energy surface. Specifically, from measurements of pure rotational transitions in the ground state $\left(\Sigma_{0}\right)$ of ArHCN, Leopold et al. [1] suggested that the complex has a global potential minimum at the linear hydrogen bonded configuration and an additional minimum at the $T$-shaped configuration. These studies combined with subsequent measurements on the excited bending states $\left(\Sigma_{1}\right.$ and $\left.\Pi_{1}\right)$ of ArHCN by Drucker et al. [6] are believed to be sufficient to reflect unambiguously virtually the entire angular coordinate $(\theta)$ along the radial minimum of the potential. An attempt [13] has

\footnotetext{
* Corresponding author. Fax: + 351-39-827703.
}

also been made to fit measured data, although no fit has thus far been reported, to an empirical function which considered all the available spectroscopic measurements. Indeed, a fit of a potential energy surface (potential function) to the available spectroscopic data alone may not even be possible due to the large uncertainties still existing in the stretching frequencies. Thus, no general consensus on the experimental potential energy surface of $\mathrm{ArHCN}$ seems to exist [10].

Theoretically, the large dimensionality (6D) of the title system and the need for using highly correlated ab initio methods and large basis sets to obtain accurate energies, makes such studies quite challenging. To our knowledge, only two ab initio studies have been reported in the literature, namely the CEPA-1 calculations of Clary et al. [9] and the MP4 calculations of Tao et al. [10]. Both sets of calculations predict the absolute minimum of the ArHCN potential energy surface to occur at linear geometries with the $\mathrm{Ar}$ atom at the $\mathrm{H}$ side of $\mathrm{HCN}$ but differ 
markedly on details concerning the variation of the energy with $\theta$. An electrostatic potential for the region close to the collinear $\mathrm{Ar}-\mathrm{HCN}$ configuration has also been presented by Klots et al. [4]. This predicts the absolute minimum to occur at $\theta=16^{\circ}$, and a barrier of $3 \mathrm{~cm}^{-1}$ to a broad linear minimum. All the above theoretical potentials have been used for spectroscopic studies with very encouraging results (see below). Of course, in spite of the level of accuracy achieved by ab initio theories, there are potential errors that cannot be eliminated without consideration of the full $6 \mathrm{D}$ potential energy surface, i.e. of the intramolecular vibrations.

We have reported a global potential energy surface [12] for the title molecule which has been obtained by summing the pair-potentials involving the $\mathrm{Ar}$ atom to a double many-body expansion (DMBE) potential function [14] for HCN. Such pair potentials have been represented by extended Hartree-Fock (EHFACE2) curves which are commonly used within the general DMBE strategy for potential energy surfaces. Thus, it has been (and will be heretofore) denoted as the ArHCN DMBE potential energy surface, although three- and four-body terms involving the Ar atom have been neglected. This fully $6 \mathrm{D}$ potential function has been employed in the same paper [12] for a detailed dynamics study of the dissociation reaction $\mathrm{Ar}+\mathrm{HCN} \rightarrow \mathrm{Ar}+\mathrm{H}+$ $\mathrm{CN}$, with the calculated thermal rate constant being found to be in fairly good agreement with the available experimental data. Unfortunately, the DMBE potential energy surface is unable to predict spectroscopic data for the ArHCN van der Waals molecule in satisfactory agreement with the available measurements. On the other hand, simple forms based on fits to accurate ab initio energies using a Legendre analysis terminated at the first few anisotropic terms can yield spectroscopic properties for ArHCN in fairly good agreement with the experimental data. One such a form has been reported by Clary et al. [9] and a more recent potential function which employs cubic splines to interpolate the calculated $(R, \theta)$ data has been published by Tao et al. [10]. However, a fit to $a b$ initio energies employing the functional form of Clary et al. [9] is easier to make than with the seminumerical potential utilized by Tao et al. [10] and hence such a form will also be used here. Unfortunately, it proved unsuccessful in fitting the data of Tao et al. [10] due to the peculiar variation of their surface with $\theta$ for a fixed radial distance and hence precluded us from attempting a similar study using their more recent ab initio information.

In summary, at the present state of knowledge, the ideal potential energy surface for the ArHCN molecule would therefore be one that combines the global DMBE form with a Legendre-type analysis (or a form similar to that used by Tao et al. [10], if one so wishes) for the $\mathrm{Ar} \cdots \mathrm{HCN}$ van der Waals interaction. In this Letter, we report such a global form by employing the energy switching (ES) scheme recently proposed by one of us [15]. This method is general and has been used to construct both singlevalued [15] and multivalued [16-18] potential energy surfaces. Moreover, we make an attempt to investigate to what extent a Legendre analysis to the $a b$ initio data of Clary et al. [9] can be consistent with the measured spectroscopic data. Given the scarcity of available experimental information on this van der Waals molecule, constrained least-squares techniques such as those reported in this Letter which combine both the accurate ab initio energies and the observed term values seem to offer a most promising route towards accurate potential energy surfaces for van der Waals molecules. The structure of the Letter is as follows. Section 2 describes the method, Section 3 presents the results and discussion and the conclusions are in Section 4.

\section{ES potential energy surface for ArHCN}

Following the ES scheme $[15,16]$, we write the ArHCN ground state potential energy surface as

$$
V_{\mathrm{ES}}(\boldsymbol{R})=f(\Delta E) V_{1}(\boldsymbol{R})+[1-f(\Delta E)] V_{2}\left(\boldsymbol{R}^{\prime}\right),
$$

where $V_{1}(\boldsymbol{R})$ is the DMBE potential energy surface [12], $V_{2}\left(\boldsymbol{R}^{\prime}\right)$ is the ab initio potential of Clary et al. [9] for the $\mathrm{Ar} \cdots \mathrm{HCN}$ van der Waals interaction, $\boldsymbol{R}$ and $\boldsymbol{R}^{\prime}$ are collective variables to be specified below, $\Delta E=E-E_{0}$ is the displacement from a reference energy and $f(\Delta E)$ is a switching function in the energy space. Following previous work $[15,16]$, this has been chosen to assume the form

$f(\Delta E)=\frac{1}{2}\left\{1+\tanh \left[\gamma_{E} \Delta E\right]\right\}$ 
where $\gamma_{E}$ is a parameter to be optimized by trialand-error for a selected reference energy. In the present work, this optimization has been done from the requirement that $V_{\mathrm{ES}}$ should give a good description of the rovibrational spectra for the ArHCN van der Waals molecule, while being free from spurious topographical features at other regions of configuration space. Such a procedure led to a value of $\gamma_{E}=0.05\left(\mathrm{~cm}^{-1}\right)^{-1}=10973 E_{\mathrm{h}}^{-1}$ for a reference energy of $E_{0}=1000 \mathrm{~cm}^{-1}$.

As stated above, $V_{1}(\boldsymbol{R})$ is the ArHCN DMBE potential energy surface, which assumes the form

$$
\begin{aligned}
V_{1}(\boldsymbol{R})= & V_{\mathrm{HCN}}\left(R_{\mathrm{CN}}, R_{\mathrm{CH}}, R_{\mathrm{NH}}\right) \\
& +\sum_{\beta=\mathrm{C}, \mathrm{N}, \mathrm{H}} V_{\mathrm{Ar} \beta}\left(R_{\mathrm{Ar} \alpha}\right),
\end{aligned}
$$

where $\boldsymbol{R}$ is the collective variable of the six internuclear distances. In turn, the pair-potentials assumed the EHFACE2-type form [19]

$$
V_{\mathrm{Ar} \beta}(R)=\lambda_{\mathrm{Ar} \beta} B_{\mathrm{HF}}(R)+V_{\mathrm{dc}}(R),
$$

where $\lambda_{\operatorname{Ar} \beta}$ are scaling parameters which have been optimized to obtain a satisfactory representation of the ArHCN van der Waals potential well. Moreover, the short-range Hartree-Fock energy has been chosen to have the Born-Mayer form

$V_{\mathrm{HF}}(R)=A \exp (-b R)$,

with the parameters $A$ and $b$ being fitted to ab initio Hartree-Fock energies [12]. In addition, the dynamical correlation energy has been modelled semiempiricaly by the damped dispersion series expansion

$V_{\mathrm{dc}}(R)=-\sum_{n=6,8,10} \chi_{n}(R) \frac{C_{n}}{R^{n}}$,

where $C_{n}$ are the dispersion energy coefficients and $\chi_{n}(R)$ are damping functions which have been described elsewhere [20],

$\chi_{n}(R)=\left[1-\exp \left(-A_{n} x-B_{n} x^{2}\right)\right]^{2}$,

where $\chi=R / \rho$ is a reduced coordinate and $\rho$ a suitably chosen scaling parameter with the dimensions of bond length ( $\left.1 \mathrm{bohr}=1 a_{0}\right)$. For two interacting atoms $\mathrm{X}$ and $\mathrm{Y}$, this assumes the form $\rho=$ $\left.2\left(\left\langle r_{\mathrm{X}}^{2}\right\rangle^{1 / 2}+\left\langle r_{\mathrm{Y}}^{2}\right\rangle\right)^{1 / 2}\right)$, with $\left\langle r_{\mathrm{X}}^{2}\right\rangle$ and $\left\langle r_{\mathrm{Y}}^{2}\right\rangle$ being the expectation value of the squared radius for the outermost electrons in atoms $\mathrm{X}$ and $\mathrm{Y}$, respectively.
In turn, $A_{n}$ and $B_{n}$ are defined by $A_{n}=\alpha_{0} n^{-\alpha 1}$ and $B_{n}=\beta_{0} \exp \left(-\beta_{1} n\right)$, respectively; $\alpha_{0}=16.36606$ $a_{0}, \alpha_{1}=0.70172, \beta_{0}=17.19338 a_{0}^{-2}$, and $\beta_{1}=$ 0.09574 are universal parameters.

To represent the Ar $\cdots \mathrm{HCN}$ van der Waals interaction accurately we have employed the form

$$
\begin{aligned}
V_{2}\left(\boldsymbol{R}^{\prime}\right)= & V_{\mathrm{HCN}}\left(R_{\mathrm{CN}}, R_{\mathrm{CH}}, R_{\mathrm{NH}}\right) \\
& +\sum_{n=0}^{6} c_{n}(R) P_{n}(\cos \theta),
\end{aligned}
$$

where the second term is an ab initio atom-rigid triatom potential function similar to that of Clary et al. [9]; $\vec{R}$ is the vector that joins the argon atom to the center of mass of the HCN molecule and $\theta$ is the angle of orientation of this vector with respect to that $(\vec{r})$ defined by the $\mathrm{HCN}$ axis. In turn, $P_{n}(\cos \theta)$ is a Legendre polynomial and

$$
c_{n}(R)=\left[\sum_{k=0}^{3} \tilde{A_{n k}} R^{k} \exp (-2 R)\right]+\chi_{6}(R) \frac{\tilde{A_{n 4}}}{R^{6}},
$$

with $\chi_{6}(R)$ being the atom-atom dispersion damping function defined in Eq. (6); $R$ is in bohr $\left(a_{0}\right)$. Moreover, $V_{\mathrm{HCN}}$ in Eq. (8) is the DMBE potential energy surface [14] for HCN, which introduces the proper dependence on the intramolecular degrees of freedom. Thus, $\boldsymbol{R}^{\prime}$ in Eq. (8) is a collective variable of only five coordinates since the van der Waals potential form assumes a rigid triatomic $\mathrm{HCN}$ molecule. In spite of this, the results reported below show that the ES approach warrants a smooth transition from the low-energy regions, where the van der Waals potential $V_{2}\left(\boldsymbol{R}^{\prime}\right)$ is valid, to the high-energy regions where $V_{1}(\mathbf{R})$ is expected to dominate and provide an acceptable representation of the true ArHCN potential energy surface. We further observe that the radial part of the van der Waals potential has the standard form of a polynomial multiplied by an exponential for the short-range term and a damped attractive $R^{-6}$ term for the long-range part. This differs from the form used by Clary et al. [9] only in the dispersion damping functions, which we have assumed to be given by Eq. (6) with the scaling parameter fixed at $\rho=17.62 a_{0}$. Thus, $\rho$ has been approximated under the assumption that $\mathrm{HCN}$ may 
be replaced by the corresponding isoelectronic atom, i.e. Si. Although this may be questionable, we have ignored the $\theta$ dependence of $\rho$. Moreover, we have jointly treated the leading coefficients of the induction and dispersion series expansions, which characterize the $\mathrm{Ar} \cdots \mathrm{HCN}$ van der Waals interaction and are known to vary as $R^{-6}$.

\section{Results and discussion}

To determine the 35 parameters in Eq. (9), we have performed three distinct fits. In fit I we have employed the standard Lavenberg-Marquardt [21] method to minimize the sum of the squared deviations and used as input data the 98 CEPA- 1 energies reported in table 2 of Ref. [9]. Table 1 gives the numerical values of the least-squares coefficients obtained in this way. Clearly, this fit gives an excellent representation of the ab initio energies: the root mean squared error is only rmsd $=0.22 \mathrm{~cm}^{-1}$ and for all fitted points is the error no larger than 1 $\mathrm{cm}^{-1}$.
Fit II consisted of a least-squares fit to the same 98 ab initio CEPA-1 energies but subject to the constraints imposed from the requirement that the deviations relative to some selected observed frequencies which are reported in Table 2 was minimal (note that our tabulated experimental frequencies differ slightly from those given in Ref. [10], which is apparently due to the use of different conversion factors from $\mathrm{Hz}$ to $\mathrm{cm}^{-1}$ ). From a technical point of view, it perhaps suffices to say that the calculation of the rovibrational energy levels of the atom-rigid linear triatom $\mathrm{Ar}-\mathrm{HCN}$ van der Waals molecule was carried out by using the Hutson [22] BOUND code, which solves the quantum mechanical bound state problem by using the coupled channel method $[23,24]$. For the calculations, the used space-fixed basis set included all rotor functions up to $j=15$, with the HCN rotational constant being taken as $1.47822 \mathrm{~cm}^{-1}$ and the reduced mass of the complex as 16.1171713 a.u. Moreover, the coupled equations have been propagated from $R_{\min }=2.8 \AA$ up to $R_{\max }=12.0 \AA$ with a step size of $0.01 \AA$. The achieved convergence is estimated to be better than

Table 1

Numerical values of the parameters in the Legendre analysis of the van der Waals potential energy function

\begin{tabular}{|c|c|c|c|c|c|c|}
\hline Fit & $n$ & $\tilde{A}_{n 0}$ & $\tilde{A}_{n 1}$ & $\tilde{A_{n 2}}$ & $\tilde{A_{n 3}}$ & $\tilde{A}_{n 4}$ \\
\hline \multirow[t]{7}{*}{ I } & 0 & 11745.326 & -5780.1721 & 1032.3531 & -59.542536 & -113.06788 \\
\hline & 1 & 6054.7165 & -3301.5824 & 645.26673 & -39.074103 & -18.655958 \\
\hline & 2 & 16318.187 & -8463.4669 & 1603.0682 & -95.812279 & -58.692529 \\
\hline & 3 & 6804.4981 & -3443.9896 & 656.15722 & -39.321323 & -18.236588 \\
\hline & 4 & 8394.1374 & -4009.2812 & 740.14154 & -43.929894 & -6.0695951 \\
\hline & 5 & 8452.1237 & -3574.9885 & 583.48330 & -32.170775 & -2.6182992 \\
\hline & 6 & 6802.4077 & -2787.2273 & 419.01926 & -21.333457 & -2.7939232 \\
\hline \multirow[t]{7}{*}{ II } & 0 & 11748.352 & -5778.6091 & 1032.2115 & -59.542591 & -114.67403 \\
\hline & 1 & 6058.2945 & -3298.0677 & 645.80489 & -39.204260 & -22.317774 \\
\hline & 2 & 16315.014 & -8466.1338 & 1603.2428 & -95.796360 & -55.729385 \\
\hline & 3 & 6801.5235 & -3447.9586 & 655.54162 & -39.179749 & -13.107113 \\
\hline & 4 & 8391.6672 & -4010.4584 & 740.01102 & -43.930869 & -2.9393666 \\
\hline & 5 & 8453.2925 & -3575.7421 & 583.24276 & -32.116822 & -2.1205591 \\
\hline & 6 & 6803.7117 & -2786.1817 & 418.98904 & -21.335325 & -4.6640358 \\
\hline \multirow[t]{7}{*}{ III } & 0 & 11748.257 & -5778.8812 & 1032.2255 & -59.542591 & -114.55528 \\
\hline & 1 & 6057.8911 & -3298.5180 & 645.64402 & -39.204260 & -22.618935 \\
\hline & 2 & 16315.701 & -8465.7118 & 1603.5433 & -95.796360 & -55.674310 \\
\hline & 3 & 6801.6222 & -3448.3143 & 655.18870 & -39.202533 & -13.318227 \\
\hline & 4 & 8391.3186 & -4010.8525 & 739.73369 & -44.015083 & -3.3593596 \\
\hline & 5 & 8453.4256 & -3575.7360 & 583.26356 & -32.086456 & -2.3740885 \\
\hline & 6 & 6803.9682 & -2785.8599 & 419.16555 & -21.297180 & -4.6972398 \\
\hline
\end{tabular}


Table 2

Calculated and observed term values (in $\mathrm{cm}^{-1}$ ) for the ground state $\Sigma_{0}$ and the lowest excited $\Sigma_{1}$ and $\Pi_{1}$ bending states of the ArHCN van der Waals molecule

\begin{tabular}{|c|c|c|c|c|c|}
\hline$J$ & $\Sigma_{0}$ & $\Sigma_{1}$ & $\Pi_{1}^{\mathrm{f}}$ & $\Pi_{1}^{\mathrm{e}}$ & Source \\
\hline \multirow[t]{6}{*}{0} & 0 & 4.538 & - & - & ES I \\
\hline & 0 & 5.490 & - & - & ES II \\
\hline & 0 & 5.420 & - & - & ES III \\
\hline & 0 & 4.332 & - & - & Ref. [9] \\
\hline & 0 & 5.050 & - & - & Ref. [10] \\
\hline & 0 & 5.500 & - & - & a \\
\hline \multirow[t]{6}{*}{1} & 0.096 & 4.650 & 7.166 & 7.167 & ES I \\
\hline & 0.098 & 5.589 & 6.142 & 6.159 & ES II \\
\hline & 0.098 & 5.524 & 6.160 & 6.173 & ES III \\
\hline & 0.097 & - & 6.727 & - & Ref. [9] \\
\hline & 0.105 & 5.174 & 5.798 & 5.801 & Ref. [10] \\
\hline & 0.107 & 5.626 & 6.138 & 6.143 & $\mathrm{a}$ \\
\hline \multirow[t]{5}{*}{2} & 0.287 & 4.872 & 7.400 & 7.405 & ES I \\
\hline & 0.295 & 5.788 & 6.370 & 6.420 & ES II \\
\hline & 0.294 & 5.733 & 6.392 & 6.429 & ES III \\
\hline & 0.317 & 5.422 & 6.063 & 6.073 & Ref. [10] \\
\hline & 0.322 & 5.880 & 6.409 & 6.422 & a \\
\hline \multirow[t]{5}{*}{3} & 0.574 & 5.206 & 7.752 & 7.762 & ES I \\
\hline & 0.589 & 6.091 & 6.712 & 6.807 & ES II \\
\hline & 0.589 & 6.050 & 6.740 & 6.810 & ES III \\
\hline & 0.631 & 5.795 & 6.462 & 6.479 & Ref. [10] \\
\hline & 0.644 & 6.260 & 6.815 & 6.839 & a \\
\hline \multirow[t]{5}{*}{4} & 0.955 & 5.652 & 8.221 & 8.237 & ES I \\
\hline & 0.982 & 6.503 & 7.167 & 7.314 & ES II \\
\hline & 0.978 & 6.476 & 7.202 & 7.313 & ES III \\
\hline & 1.050 & 6.295 & 6.992 & 7.018 & Ref. [10] \\
\hline & 1.072 & 6.771 & 7.356 & 7.392 & a \\
\hline \multirow[t]{5}{*}{5} & 1.433 & 6.208 & 8.806 & 8.830 & ES I \\
\hline & 1.472 & 7.025 & 7.735 & 7.939 & ES II \\
\hline & 1.466 & 7.014 & 7.780 & 7.937 & ES III \\
\hline & 1.574 & 6.923 & 7.655 & 7.690 & Ref. [10] \\
\hline & 1.606 & 7.412 & 8.030 & 8.081 & a \\
\hline \multirow[t]{5}{*}{6} & 2.044 & 6.876 & 9.540 & 9.507 & ES I \\
\hline & 2.059 & 7.659 & 8.416 & 8.681 & ES II \\
\hline & 2.051 & 7.664 & 8.471 & 8.678 & ES III \\
\hline & 2.200 & 7.680 & 8.449 & 8.492 & Ref. [10] \\
\hline & 2.246 & 8.185 & & & a \\
\hline
\end{tabular}

${ }^{a}$ Experimental, Refs. [2,3,6].

$0.001 \mathrm{~cm}^{-1}$ for energy levels up to a total angular momentum of $J=6$, which concerns us most in the present work. Returning to the least-squares approach, the minimization of the sum of squared deviations (which includes those relative to the 5 selected experimental rovibrational frequencies, all for $J=0,1$ ) has been carried out using the Metropo- lis Monte Carlo method. Thus, the BOUND code has been treated as a subroutine which is called by the main least-squares program at the various iteration steps during the minimization procedure. A weight of $10^{3}$ has been assigned to each fitted frequency during the minimization procedure whereas every $a b$ initio point carried a unit weight. It should be emphasized that, except for the $J=0,1$ frequencies, all others have not been adjusted and hence may serve as data for testing the quality of the fitting procedure. Such a procedure led to a decrease of the rmsd referring to the frequencies, which diminished from $0.89 \mathrm{~cm}^{-1}$ for fit I to a value of $0.11 \mathrm{~cm}^{-1}$. To improve this result further, the weights of the fitted frequencies were then raised to $5 \times 10^{4}$, and the minimization allowed to continue until the rmsd of the calculated frequencies attained a value $0.02 \mathrm{~cm}^{-1}$. As expected, the quality of the fit with respect to the ab initio points deteriorated slightly, with the rmsd = $2.39 \mathrm{~cm}^{-1}$ in fit II.

While testing the function obtained in fit II, we have observed that the experimental [6] properties $\left\langle P_{1}(\cos \theta)\right\rangle$ and $\left\langle P_{2}(\cos \theta)\right\rangle$ for the $\Sigma_{1}$ and $\Pi_{1}$ states were not well reproduced. Such properties characterize the angular $\theta$ distribution of the rovibrational wavefunctions and hence are believed to be sensitive to the potential anisotropy [10]. This negative result led us to conclude that a fit of the ab initio energies of Clary et al. [9], subject only to the constraints provided by the above-mentioned 5 frequencies, was not sufficient to obtain an accurate description of the ArHCN interaction potential. In an attempt to remedy this problem, fit III included as a further constraint that provided by the experimental $\left\langle P_{2}(\cos \theta)\right\rangle$ values. The corresponding deviations relative to this property have then been assigned a least-squares weight of $5 \times 10^{5}$, while all other fitted data carried the weights assigned in fit II. Although some improvement has been achieved, the procedure could not proceed without leading to further deterioration in the quality of the fit with respect to the $a b$ initio energies and frequencies; the separate rmsd with respect to the ab initio energies, frequencies and $\left\langle P_{2}(\cos \theta)\right\rangle$ properties are in fit III 4.18, 0.05, and $0.09 \mathrm{~cm}^{-1}$, respectively. Since none of the fits may be judged to be exempt from some deficiency, we give the values of the least-squares coefficients in Table 1 for fits II and III also. 

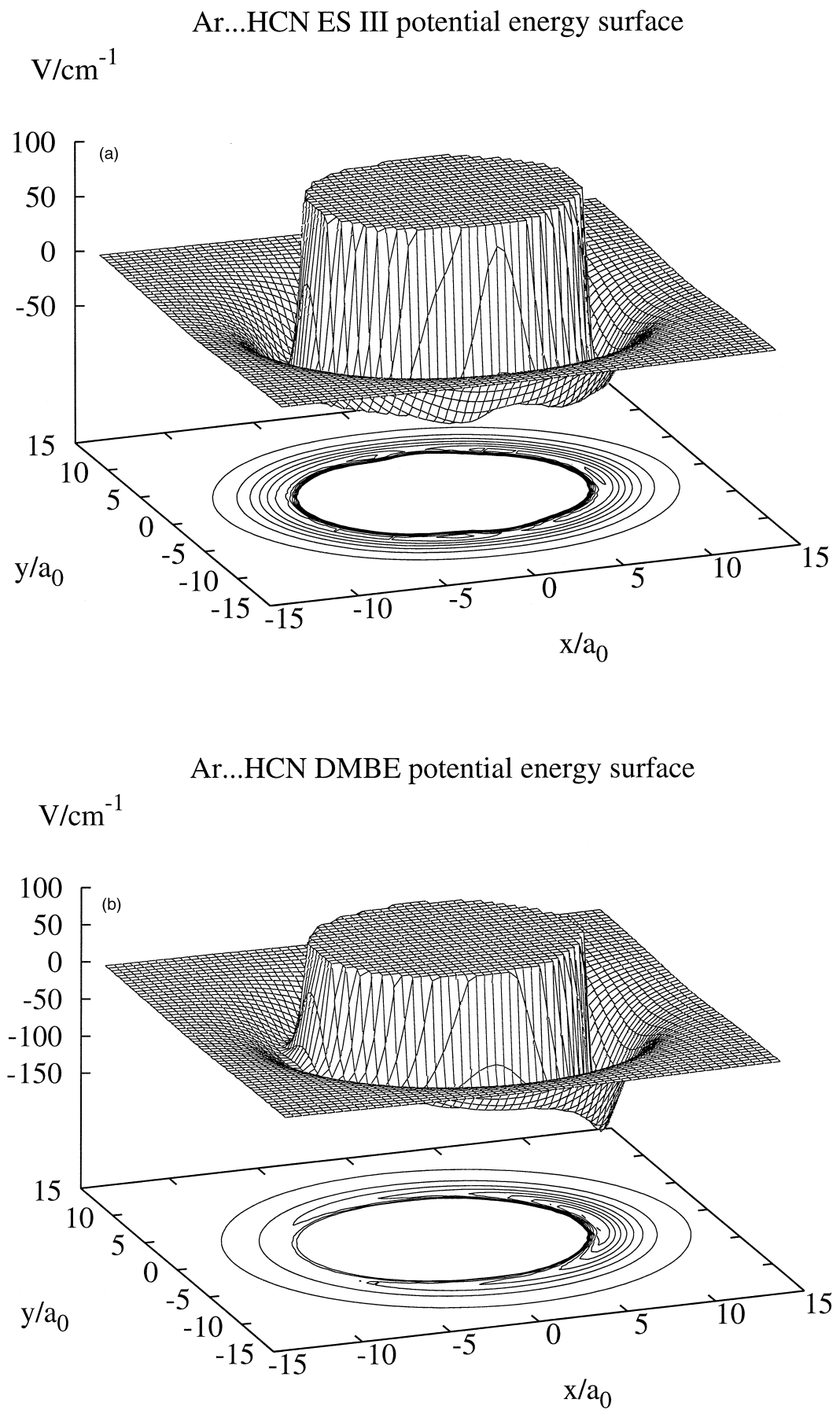

Fig. 1. Perspective view/contour plot of the ArHCN potential energy surface for an Ar atom moving around an $\mathrm{HCN}$ molecule fixed at its linear equilibrium geometry: (a) DMBE potential energy surface 12; and (b) ES III (this Letter). 
Using the above three fits to the van der Waals part of the potential energy surface, we then obtained the corresponding ES potential energy surfaces. Because all ES potential functions have rather similar topographies, we show for comparison in Fig. 1 only a perspective view/contour plot of the ArHCN DMBE (in Fig. 1a) and ES III (in Fig. 1b) potential energy surfaces for an Ar atom moving around an $\mathrm{HCN}$ molecule fixed at its linear equilibrium geometry. As can be seen from Figs. 1 and 2, the ES III potential energy surface shows a minimum interaction energy which is slightly shallower than that predicted by the ab initio calculations and ES I, namely $V_{\mathrm{m}}=-85.0 \mathrm{~cm}^{-1}$ at $R_{\mathrm{m}}=8.75 a_{0}$ and $\theta=0^{\circ}$. Indeed, in comparison with the corresponding DMBE plot [12], the ES III potential function (as well as ES I and ES II) of the present work show considerable differences concerning the well depth and anisotropy of the van der Waals well. This is especially visible from Fig. 2, which compares the DMBE [12] and ES III potential energy surfaces for cuts on Fig. 1 corresponding to $\theta=0^{\circ}$ (Fig. 2a), $\theta=90^{\circ}$ (Fig. 2b), and $\theta=180^{\circ}$ (Fig. 2c). These plots serve also to illustrates how the ES approach works. Clearly, the ES potential forms can be quite smooth if $E_{0}$ and $\gamma_{E}$ in Eq. (2) are adequately selected. Finally, it should be noted that the ES potential functions are very similar in construction to those reported by Clary et al. [9] in what concerns the well depth and location of the van der Waals minimum, which is somewhat shallower than that predicted by Tao et al. [10] $\left(V_{\mathrm{m}}=-135.9 \mathrm{~cm}^{-1}\right.$ at $R_{\mathrm{m}}=8.62 \quad a_{0}$ and $\theta=0^{\circ}$ ). Moreover, besides the barrier and minima for bent geometries, all ES surfaces predict a very shallow minimum at the $N$ side. All such attributes so far have no experimental confirmation.

Fig. 3 compares the approximate minimum energy path (which has been obtained by calculating the minimum energy along rays for different values of $\theta$ ) of the three ES potential energy surfaces for the variation with the $\theta$ angle. Clearly, the effect of having included the experimental frequencies and $\left\langle P_{2}(\cos \theta)\right\rangle$ properties on the least-squares fits had important consequences on the topography of the potential energy surface, namely on the high and width of the barrier which separates the two relative minima at $\theta \sim 60^{\circ}$ and $\sim 110^{\circ}$. Moreover, although
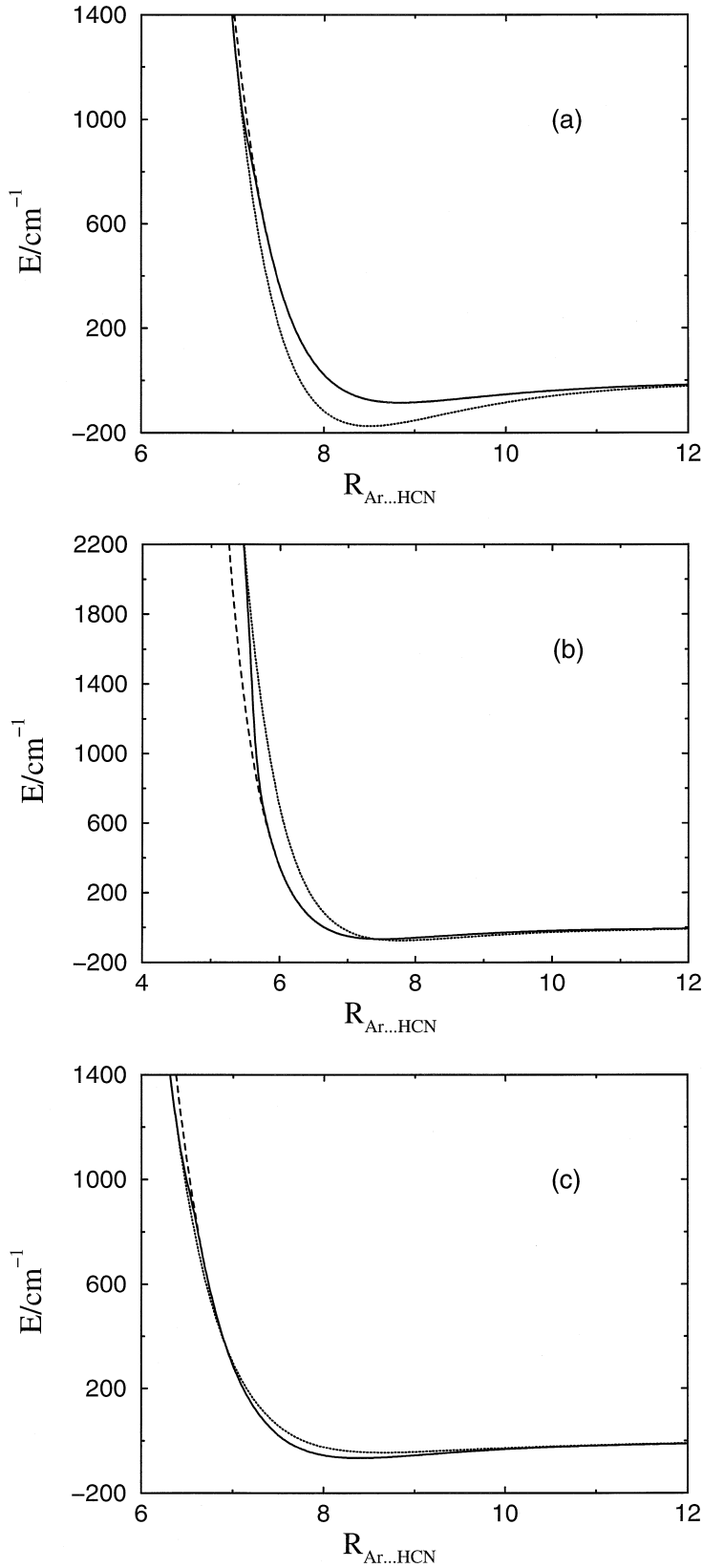

Fig. 2. Comparison of the DMBE [12], and ES III potential energy surfaces for cuts in Fig. 1 corresponding to: (a) $\theta=0^{\circ}$; (b) $\theta=90^{\circ}$; and (c) $\theta=180^{\circ}$.

the values of $\left\langle P_{1}(\cos \theta)\right\rangle$ and $\left\langle P_{2}(\cos \theta)\right\rangle$ are reasonably well described by all ES potential energy surfaces, only ES III is capable of describing them moderately well for the lowest $\Sigma_{1}$ and $\Pi_{1}$ bending 


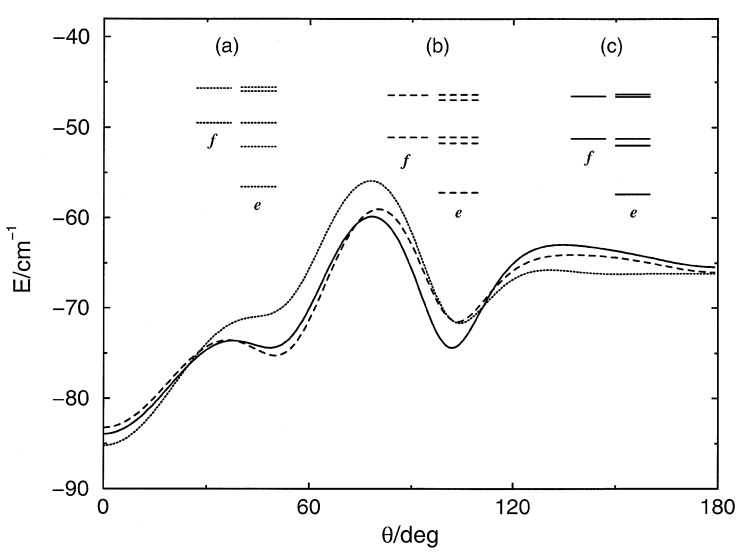

Fig. 3. Comparison of the approximate minimum energy paths for Ar moving around an $\mathrm{HCN}$ molecule fixed at its linear equilibrium geometry: dotted line, ES I; dashed line, ES II; solid line, ES III. Also shown on the plot are the first few calculated vibrational levels of even (e) and odd (f) parity.

states of ArHCN. Indeed, the topographical features occurring between $60^{\circ}$ and $120^{\circ}$ may have an important role on the extent of tunnelling and hence explain the different spectroscopic properties of three otherwise rather similar ES potential energy surfaces. This seems to suggest that the $\theta$-dependence of the true potential energy surface may have to deviate from that of the original ab initio curve of Clary et al. [9] before further progress can be made, a conclusion which supports to some extent that of Tao et al. [10]. Thus, no further attempt at improvement was judged to be warranted at this stage.

A quantitative analysis of the various ES potential energy surfaces is provided in Tables 2 and 3 . Specifically, Table 2 compares the calculated frequencies with the experimental ones, and other theoretical results $[9,10]$. The agreement with experiment is seen to be good, in particular for the nonfitted frequencies corresponding to $J=2-6$. In fact, the calculated rmsd for all frequencies reported in Table 2 are $0.18 \mathrm{~cm}^{-1}$ for the ES II and ES III potential energy surfaces, which compare with the values of $0.35 \mathrm{~cm}^{-1}$ obtained from the results of Tao et al. [10] and $0.86 \mathrm{~cm}^{-1}$ from the ES I potential function. We further observe that the splittings of the $\Pi_{1}$ states in ES II and ES III are somewhat too large in comparison with those predicted by the ab initio data (i.e., ES I) and also the experimental measurements. As pointed out in the previous paragraph, this may be attributed to a narrowing of the barrier, a topographical feature that can be an artifact and hence deserves further investigation.

Finally, we remark that the calculated ES III stretching frequency is $24.50 \mathrm{~cm}^{-1}$ (this attribute is essentially identical for all ES potential energy surfaces reported in this Letter), which is similar to the value calculated by Clary et al. [9] of $23.85 \mathrm{~cm}^{-1}$. Thus, it is somewhat too small when compared with the experimental estimates of Fraser and Pine [3] (33 $\left.\mathrm{cm}^{-1}\right)$ and Drucker et al. [6] $\left(36.1 \mathrm{~cm}^{-1}\right)$, although it overestimates that of Leopold et al. [1] which is only $10 \mathrm{~cm}^{-1}$. Unfortunately, Tao et al. [10] do not give the stretching frequency, which prevents a comparison with this attribute for their interaction potential energy surface.

To conclude this section, we comment briefly on studies of the reaction $\mathrm{Ar}+\mathrm{HCN} \rightarrow \mathrm{Ar}+\mathrm{H}+\mathrm{CN}$ and $\mathrm{Ar}+\mathrm{HCN}$ energy transfer process which have been carried out using the quasiclassical trajectory (QCT) method [25] and the ES potential energy surfaces from the present work. Because such calculations have duplicated in all respects those previously reported using the ArHCN DMBE potential energy surface, no details are deemed necessary to be given here. In fact, as might have been anticipated, only minor deviations have been observed at extremely low collisional energies, although they lie

Table 3

Calculated and observed properties for the ground state $\Sigma_{0}$ and the lowest excited $\Sigma_{1}$ and $\Pi_{1}$ bending states of the ArHCN van der Waals molecule

\begin{tabular}{llrrl}
\hline & $\Sigma_{0}$ & \multicolumn{1}{l}{$\Sigma_{1}$} & \multicolumn{1}{l}{$\Pi_{1}$} & Source \\
\hline$\left\langle P_{1}(\cos \theta)\right\rangle$ & 0.838 & -0.484 & -0.070 & ES I \\
& 0.778 & -0.275 & 0.278 & ES II \\
& 0.787 & -0.194 & 0.208 & ES III \\
& 0.823 & -0.473 & -0.021 & Ref. [9] \\
& 0.853 & -0.154 & 0.100 & Ref. [10] \\
$\left\langle P_{2}(\cos \theta)\right\rangle$ & 0.875 & -0.175 & 0.091 & a \\
& 0.611 & 0.102 & -0.136 & ES I \\
& 0.495 & 0.030 & -0.071 & ES II \\
& 0.515 & -0.053 & -0.120 & ES III \\
& 0.589 & 0.121 & -0.137 & Ref. [9] \\
& 0.578 & -0.139 & -0.208 & Ref. [10] \\
& 0.607 & -0.175 & -0.192 & a \\
\hline
\end{tabular}

${ }^{\mathrm{a}}$ Experimental, Refs. [5,6]. 
within the statistical uncertainty of the calculations and hence have no physical justification. Thus, we conclude that the ES I to ES III potential energy surfaces have the same dynamics and kinetic attributes as the DMBE potential function for ArHCN, except perhaps for purely classical calculations (i.e., without consideration of zero-point energies) at very low collisional energies.

\section{Concluding remarks}

We have reported realistic global potential energy surfaces for ArHCN by the ES method. They have been obtained by merging a DMBE potential energy surface, which behaves correctly at all dissociation limits, with Legendre type analysis, which reproduces the properties of the ArHCN van der Waals molecule. Their quality has also been assessed through calculations of rovibrational levels for the ArHCN van der Waals molecule and reactive dynamics calculations. Although we emphasize that significant errors may still persist in the description of the van der Waals interaction, the ES III potential energy surface reported in this Letter is, to our knowledge, the only existing potential energy surface capable of reproducing accurately both the kinetics of the reaction $\mathrm{Ar}+\mathrm{HCN} \rightarrow \mathrm{Ar}+\mathrm{H}+\mathrm{CN}$ and the rovibrational spectroscopy of the ArHCN molecule. Given the orders of magnitude difference between the energies relevant for studying such processes, the ES method has also revealed itself as the only affordable scheme capable of yielding an accurate potential energy surface which encompasses both such energy regimes.

\section{Acknowledgements}

This work has the support of Fundação para a Ciência e Tecnologia, Portugal, under programmes PRAXIS XXI and FEDER (Contract 2/2.1/
QUI/408/94). PAJG carried out this work as part of his graduation thesis in chemistry.

\section{References}

[1] K.R. Leopold, G.T. Fraser, F.J. Lin, D.D. Nelson Jr., W. Klemperer, J. Chem. Phys. 81 (1984) 4922.

[2] R.E. Bumgarner, G.A. Blake, Chem. Phys. Lett. 161 (1989) 308.

[3] G.T. Fraser, A.S. Pine, J. Chem. Phys. 91 (1989) 3319

[4] T.D. Klots, C.E. Dykstra, H.S. Gutowsky, J. Chem. Phys. 90 (1989) 30.

[5] A.L. Cooksy, S. Drucker, J. Faeder, C.A. Gottlieb, W. Klemperer, J. Chem. Phys. 95 (1991) 3017.

[6] S. Drucker, A.L. Cooksy, W. Klemperer, J. Chem. Phys. 98 (1993) 5158.

[7] E. Arunan, T. Emilsson, H.S. Gutowski, J. Chem. Phys. 103 (1995) 493

[8] K. Uemura, A. Hara, K. Tanaka, J. Chem. Phys. 104 (1996) 9747.

[9] D.C. Clary, C.E. Dateo, T. Stoecklin, J. Chem. Phys. 93 (1990) 7666.

[10] F.-M. Tao, S. Drucker, W. Klemperer, J. Chem. Phys. 102 (1995) 7289.

[11] J.M. Bowman, D.A. Padmavathi, Mol. Phys. 88 (1996) 21.

[12] S.P.J. Rodrigues, A.J.C. Varandas, J. Phys. Chem. 102 (1998) 6266.

[13] D. Yaron, W. Klemperer, J. Chem. Phys. 95 (1991) 1907.

[14] A.J.C. Varandas, S.P.J. Rodrigues, J. Chem. Phys. 106 (1997) 9647.

[15] A.J.C. Varandas, J. Chem. Phys. 105 (1996) 3524.

[16] A.J.C. Varandas, J. Chem. Phys. 107 (1997) 867.

[17] A.J.C. Varandas, A.I. Voronin, P.J.S.B. Caridade, J. Chem. Phys. 108 (1998) 7623.

[18] R. Prosmiti, O.L. Polyansky, J. Tennyson, Chem. Phys. Lett. 273 (1997) 107.

[19] A.J.C. Varandas, Adv. Chem. Phys. 74 (1988) 255.

[20] A.J.C. Varandas, Mol. Phys. 60 (1987) 527.

[21] W.H. Press, B.P. Flannery, S.A. Teukolsky, W.T. Vetterling, Numerical Recipes, Cambridge University Press, London, 1986.

[22] J.M. Hutson, BOUND computer code, Version 5, distributed by Collaborative Computational Project No. 6 of the Science and Engineering Research Council, UK, 1993.

[23] B.R. Johnson, J. Chem. Phys. 69 (1978) 4678.

[24] J.M. Hutson, Comput. Phys. Commun. 84 (1994) 1.

[25] D.G. Truhlar, J.T. Muckerman, in: R.B. Bernstein (Ed.), Atom-Molecule Collision Theory, Plenum, New York, 1979, p. 505 . 